\title{
The Dynamics on Hammer with Three Freedoms and Friction Vibration by Lagrange Equation in Robotic Arm
}

run xu ( $\square$ xurun1206@163.com )

Original Article

Keywords: Dynamic Equation, hammering , Robotic Arm, Lagrange Equation, three freedoms

Posted Date: June 30th, 2020

DOI: https://doi.org/10.21203/rs.3.rs-37335/v1

License: (c) (i) This work is licensed under a Creative Commons Attribution 4.0 International License.

Read Full License 


\section{The Dynamics on Hammer with Three Freedoms and Friction Vibration by Lagrange Equation in Robotic Arm}

\section{Run Xu}

Gyeongsang National University, Metallurgical Engineering Department, Gyeongsang nam-do , Chinju 52828, Korea

Abstract: the dynamic equation of three freedoms and friction impulsion vibration is established hammer in robotic arm by Larange equation. It is based on one of two freedoms. It is found that the first and second items is long and others is concise which is found in kinetic equation in this study.

Keywords Dynamic Equation, hammering, Robotic Arm, Lagrange Equation, three freedoms

\section{Introduction}

In modern industry the activity of robotic arm is high as a automatic device. It is an important branch for the Robot. Its feature has been completing anticipated working task by program. It is a automatic device for robot technological field to be gained the most practical applications that is in industry manufacture, medical healing, military, semi-conductive manufacture and space exploring. Its structure and property have an advantage of human and robot respectively especial in exhibiting human intelligence and adaption. Its precision and capability in all kinds of environment is excellent so that it has wide prospects in each field of economy. The punching destruction is often used in destruction applications. It shall be studied detail that dynamic equation is established to grasp each parameters to wield its virtual use value. Because it has main two freedoms one is rotation and the other is punch its this equation will be established according to these features. The each dynamic equation is plotted then substitute into Lagrange equation to search for the dynamic equation to be established. ${ }^{[1]}$ Because it has five parts to move and hammering below equation is to be solved according to each parts movement. After establishing this equation it can be analyzed by related parameters to optimum and cost decreasing. The length and mass of components and position will be control parameters. ${ }^{[2]}$ So in this paper thees parameters will be further discussed to look for cost decreasing. It is hopeful to assist designer and related teacher in studying further at factory and university. 
2 Modeling and establishing dynamic equation

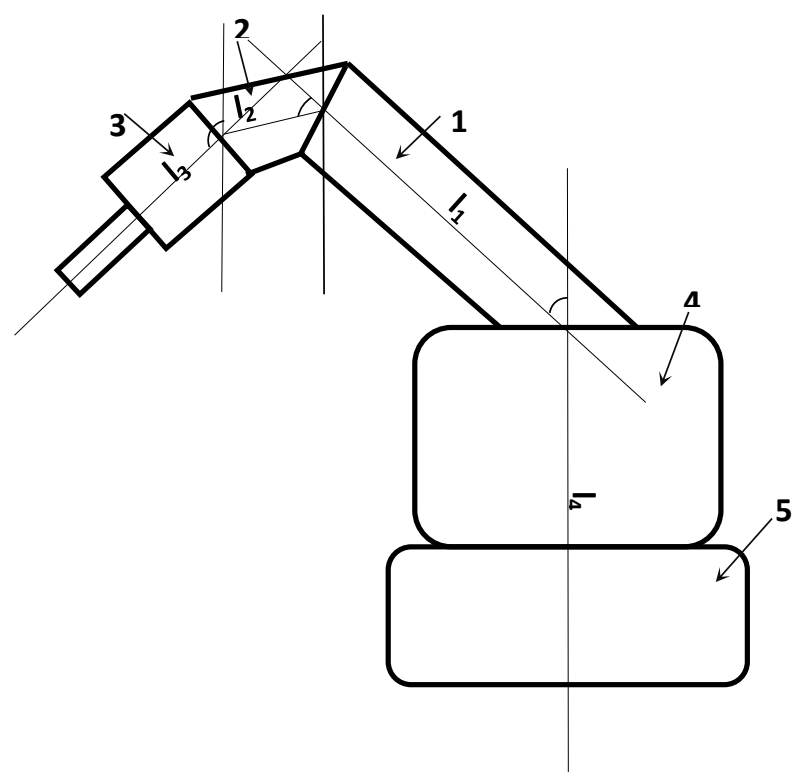


Figure 1 construction schematic of mechanical arm in series in robot

3-hand part; 2-wrist part;1-arm part; 4-waist part; 5-two crawling wheel

In Figure 1 there are five freedoms in mechanical arm that name as $1 \sim 3$. Meantime there are two other ones call $4 \& 5$.

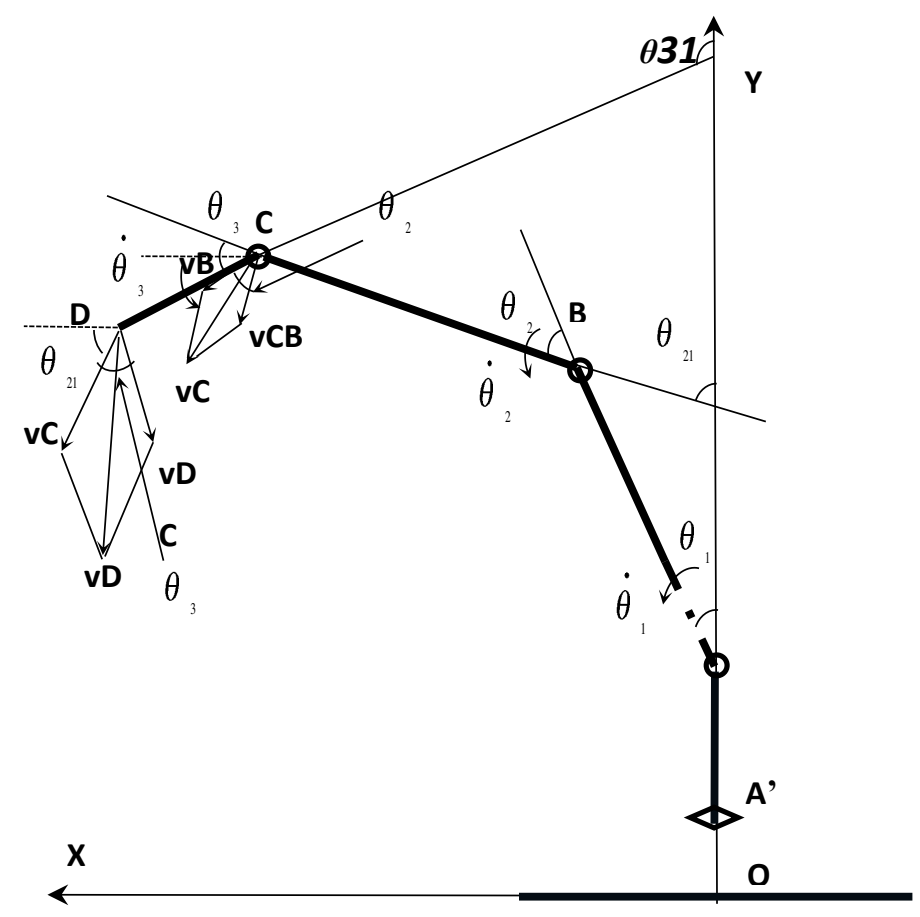

A

Figure 2 principle schematic of mechanical arm in series in robot

$$
\text { Here } \theta_{21}=360^{\circ}-\left(\theta_{1}+\theta_{2}\right) \text {. }
$$

System kinetic energy is 


$$
E_{k}=\frac{1}{2} \sum_{i}^{n}\left(m_{1} v_{1}^{2}+m_{2} v_{2}^{2}+m_{3} v_{3}^{2}\right)
$$

Here $m_{i}$ : mass of i component ; $J_{s i}$ : rotary inertia of i component relative to center of mass; $v_{s}$ : center of mass in i component; $\omega_{i}$ : angular velocity in i component; $v_{1}, v_{2}$ and $v_{3}$ is 1,2 and 3 velocities respectively.

$v_{D}=\sqrt{\dot{X_{D}^{2}+Y_{D}^{2}}}$

From Figure 2 it is known that position coordinate below

$$
\left\{\begin{array}{l}
X_{D}=\vec{l}_{1} \sin \vartheta_{1}+\vec{l}_{2} \sin \left(\theta_{1}+\theta_{2}\right)+\vec{l}_{3} \sin \left(\theta_{1}+\theta_{3}+\theta_{3}\right) \\
Y_{D}=\left(\vec{l}_{1}+\vec{l}_{4}\right) \cos \theta_{1}+\left(\vec{l}_{2}+\vec{l}_{4}\right) \cos \left(\theta_{1}+\theta_{2}\right)+\left(\vec{l}_{3}+\vec{l}_{4}\right) \cos \left(\theta_{1}+\theta_{2}+\theta_{3}\right)
\end{array}\right.
$$

Derivating the equations we gain the $\dot{X}_{c}, \dot{Y}_{c}$ and $\dot{X}_{3}$ velocity in hand , $\dot{\theta}_{1}, \dot{\theta}_{2}$ and $\dot{\theta}_{3}$ one in joints. Suppose that the acceleration is $\ddot{\theta}_{1}, \ddot{\theta}_{2}$ and $\ddot{\theta}_{2}$ and the angular acceleration is $\ddot{\omega}_{1}, \ddot{\omega}_{2}$ and $\ddot{\omega}$, in joints.

$$
\left\{\begin{array}{l}
\dot{X}_{\mathrm{D}}=\dot{\theta}_{1} \vec{l}_{1} \cos \theta_{1}+\left(\dot{\theta}_{1}+\dot{\theta}_{2}\right) \vec{l}_{2} \cos \left(\theta_{1}+\theta_{2}\right)+\left(\dot{\theta}_{1}+\dot{\theta}_{2}+\dot{\theta}_{3}\right) \vec{l}_{3} \cos \left(\theta_{1}+\theta_{2}+\theta_{3}\right) \\
\left.\dot{Y}_{\mathrm{D}}=\dot{\theta}_{1}\left(\vec{l}_{1}+\vec{l}_{4}\right) \sin \theta_{1}+\dot{\theta}_{1}+\dot{\theta}_{2}\right)\left(\vec{l}_{2}+\vec{l}_{4}\right) \sin \left(\theta_{1}+\theta_{2}\right)+\left(\dot{\theta}_{1}+\dot{\theta}_{2}+\dot{\theta}_{3}\right)\left(\vec{l}_{3}+\vec{l}_{4}\right) \sin \left(\theta_{1}+\theta_{2}+\theta_{3}\right)
\end{array}\right.
$$

$v_{B}, v_{C}$ and $v_{D}$ is $\mathrm{B}, \mathrm{C}$ and $\mathrm{D}$ velocities respectively. So $\mathrm{D}$ point velocity is

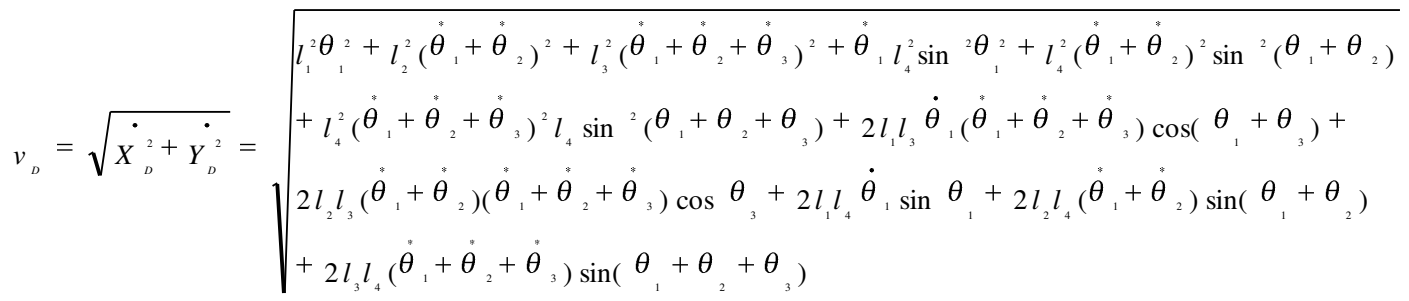

C point velocity is 
$v_{c}=\sqrt{\dot{X}_{c}^{2}+\dot{Y}_{c}^{2}}=\sqrt{\left.l_{1}^{2} \theta_{1}^{2}+l_{2}^{2} \dot{\theta}_{1}+\dot{\theta}_{2}\right)^{2}-2 l_{1} l_{2} \dot{\theta}_{1}\left(\dot{\theta}_{1}+\dot{\theta}_{2}\right) \cos \theta}$

$v_{s}=\vec{l}_{1} \dot{\theta}_{1}$

Substituting two equations above to equation below

$E_{K}=\frac{1}{2} \vec{l}_{1}\left(\vec{l}_{1}+\vec{l}_{4}+\vec{l}_{5}\right)\left(m_{1}+m_{2}+m_{3}\right)_{1} \theta_{1}^{2}+\frac{1}{2} \vec{l}_{2}\left(\vec{l}_{2}+\vec{l}_{4}+\vec{l}_{5}\right) m_{2}\left(\theta_{1}+\theta_{2}\right)^{2}+\frac{1}{2} \vec{l}_{2}\left(\vec{l}_{2}+\vec{l}_{4}+\vec{l}_{5}\right) m_{2}\left(\dot{\theta}_{1}+\dot{\theta}_{2}\right)^{2}$ $+\frac{1}{2} \vec{l}_{3}\left(\vec{l}_{3}+\vec{l}_{4}+\vec{l}_{5}\right) m_{3}\left(\theta_{1}+\theta_{2}+\theta_{3}\right)^{2}+2 \vec{l}_{4}^{2} m_{3} \dot{\theta}_{1} \sin ^{2} \theta_{2}+\vec{l}_{4}^{2} m_{3} \dot{\theta}_{1}\left(\dot{\theta}_{1}+\dot{\theta}_{2}\right)^{2} \sin { }^{2}\left(\theta_{1}+\theta_{2}\right)+\vec{l}_{4}^{2} m_{3}\left(\dot{\theta}_{1}+\dot{\theta}_{2}\right.$ $\left.\left.\left.+\dot{\theta}_{3}\right)^{2} \sin ^{2}\left(\theta_{1}+\theta_{2}+\theta_{3}\right)+2 \vec{l}_{1} \vec{l}_{2} m_{2} \dot{\theta}_{1} \dot{\theta}_{1}+\dot{\theta}_{2}\right) \cos \theta_{2}+\vec{l}_{1} \vec{l}_{2} m_{3} \dot{\theta}_{1} \dot{\theta}_{1}+\dot{\theta}_{2}+\dot{\theta}_{3}\right) \cos \left(\theta_{1}+\theta_{2}\right)+\vec{l}_{2} \vec{l}_{3} m_{3}\left(\dot{\theta}_{1}\right.$ $\left.+\dot{\theta}_{2}\right)\left(\dot{\theta}_{1}+\dot{\theta}_{2}+\dot{\theta}_{3}\right) \cos _{3}+\frac{1}{2}\left(\vec{l}_{4}+\vec{l}_{5}\right)\left(m_{1}+m_{2}+m_{3}\right) \theta_{1}^{2}+\frac{1}{2}\left(\vec{l}_{4}+\vec{l}_{5}\right) m_{2}\left(\theta_{1}+\theta_{2}\right)^{2}+\frac{1}{2}\left(\vec{l}_{4}+\vec{l}_{5}\right) m_{2}\left(\dot{\theta}_{1}+\right.$ $\left.\left.\left.\left.\dot{\theta}_{2}\right)^{2}+2 \vec{l}_{1} \vec{l}_{4} m_{3} \dot{\theta}_{1} \sin \theta_{1}+\theta_{2}\right)+2 \vec{l}_{1} \vec{l}_{4} m_{3}\left(\dot{\theta}_{1}+\dot{\theta}_{2}\right) \sin \theta_{1}+\theta_{2}\right)+2 \vec{l}_{3} \vec{l}_{4} m_{3}\left(\dot{\theta}_{1}+\dot{\theta}_{2}+\dot{\theta}_{3}\right) \sin \theta_{1}+\theta_{2}+\theta_{3}\right)$

\section{Here}

$$
\begin{aligned}
& \frac{\partial E_{K}}{\partial \dot{\theta}_{1}}=\left(\dot{\theta}_{1}+\dot{\theta}_{2}\right) \vec{l}_{2} m_{2}+2 \vec{l}_{4} m_{3} \sin { }^{2} \theta+2\left(\dot{\theta}_{1}+\dot{\theta}_{2}\right)^{2} \vec{l}_{4} m_{3} \sin ^{2}\left(\theta_{1}+\theta_{2}\right)+2 \\
& \dot{\theta}_{1}\left(\dot{\theta}_{1}+\dot{\theta}_{2}\right) \vec{l}_{4}^{2} m_{3} \sin ^{2}\left(\theta_{1}+\theta_{2}\right)+2 \vec{l}_{4} m_{3} \sin ^{2}\left(\theta_{1}+\theta_{2}+\theta_{3}\right)+2\left(\dot{\theta}_{1}+\dot{\theta}_{2}\right) \vec{l}_{1} \vec{l}_{2} \\
& m_{2} \cos \theta_{2}+2 \dot{\theta}_{1} \vec{l}_{1} \vec{l}_{2} m_{2} \cos \theta_{2}+\dot{\theta}_{1_{1}} \vec{l}_{1} \vec{l}_{2} m_{3} \cos \left(\theta_{1}+\theta_{2}\right)+\vec{l}_{1} \vec{l}_{2} m_{3}\left(\dot{\theta}_{1}+\dot{\theta}_{2}+\right. \\
& \left.\dot{\theta}_{3}\right) \cos \left(\theta_{1}+\theta_{2}+\theta_{3}\right)+\vec{l}_{2} m_{3}\left(\dot{\theta}_{1}+\dot{\theta}_{2}\right) \cos \theta_{3}+\vec{l}_{2} \vec{l}_{3} m_{3}\left(\dot{\theta}_{1}+\dot{\theta}_{2}+\dot{\theta}_{3}\right) \cos \theta_{3} \\
& +2 \vec{l}_{1} \vec{l}_{4} m_{3} \sin \left(\theta_{1}+\theta_{2}\right)+2 \vec{l}_{1} \vec{l}_{4} m_{3} \sin \left(\theta_{1}+\theta_{2}\right)+2 \vec{l}_{1} \vec{l}_{4} m_{3} \cos \left(\theta_{1}+\theta_{2}+\theta_{3}\right)
\end{aligned}
$$

(9)

$$
\begin{aligned}
& \frac{\partial_{E_{K}}}{\partial \dot{\theta}_{2}} \vec{l}_{2} m_{2}\left(\dot{\theta}_{1}+\dot{\theta}_{2}\right)+\vec{l}_{4} m_{3}\left(\dot{\theta}_{1}+\dot{\theta}_{2}\right)^{2} \sin { }^{2}\left(\theta_{1}+\theta_{2}\right)+2 \vec{l}_{4} m_{3} \dot{\theta}_{1}\left(\dot{\theta}_{1}+\dot{\theta}_{2}\right) \sin \\
& { }^{2}\left(\theta_{1}+\theta_{2}\right)+2 \vec{l}_{4} m_{3}\left(\dot{\theta}_{1}+\dot{\theta}_{2}+\dot{\theta}_{3}\right) \sin { }^{2}\left(\theta_{1}+\theta_{2}\right)+2 \vec{l}_{1} \vec{l}_{2} m_{2} \dot{\theta}_{1} \cos \theta_{2}+\vec{l}_{1} \vec{l}_{2} m_{2} \\
& \left(\dot{\theta}_{1}+\dot{\theta}_{2}\right) \cos \theta_{2}+\vec{l}_{2} \vec{l}_{3} m_{3}\left(\dot{\theta}_{1}+\dot{\theta}_{2}+\dot{\theta}_{2}\right)+\cos \left(\theta_{1}+\theta_{2}\right)+\vec{l}_{2} \vec{l}_{3} m_{3}\left(\dot{\theta}_{1}+\dot{\theta}_{2}+\dot{\theta}_{3}\right) \\
& \cos \theta_{3}+\vec{l}_{2} \vec{l}_{3} m_{3}\left(\dot{\theta}_{1}+\dot{\theta}_{2}\right) \cos \theta_{3}+2 \vec{l}_{1} \vec{l}_{4} m_{3} \sin \left(\theta_{1}+\theta_{2}\right)+2 \vec{l}_{1} \vec{l}_{4} m_{3} \sin \left(\theta_{1}+\theta_{2}\right. \\
& \left.+\theta_{3}\right)
\end{aligned}
$$


$\frac{\partial E_{k}}{\partial \dot{\theta}_{3}}=\vec{l}_{1} \vec{l}_{2} m_{3} \dot{\theta}_{1} \cos \left(\theta_{1}+\theta_{2}+\theta_{3}\right)+2 \vec{l}_{4} m_{3}\left(\dot{\theta}_{1}+\dot{\theta}_{2}+\dot{\theta}_{3}\right) \sin ^{2}\left(\theta_{1}+\theta_{2}+\right.$

$\left.\theta_{3}\right)+\vec{l}_{1} \vec{l}_{2} m_{3}\left(\dot{\theta}_{1}+\dot{\theta}_{2}+\dot{\theta}_{3}\right) \cos \left(\theta_{1}+\theta_{2}\right)+\vec{l}_{1} \vec{l}_{2} m_{3} \dot{\theta}_{1} \cos \left(\theta_{1}+\theta_{2}\right)+\vec{l}_{2} \vec{l}_{3} m$ $\left.{ }_{3} \dot{\theta}_{1}+\dot{\theta}_{2}\right) \cos \theta_{3}+\vec{l}_{1} \vec{l}_{2} m_{3} \dot{\theta}_{1} \cos \left(\theta_{1}+\theta_{2}\right)+\vec{l}_{2} \vec{l}_{3} m_{3}\left(\dot{\theta}_{1}+\dot{\theta}_{3}\right) \cos \theta_{3}+2 \vec{l}_{1} \vec{l}_{4}$

$m_{3} \cos \left(\theta_{1}+\theta_{2}+\theta_{3}\right)$

(11)

And

$\frac{d}{d t}\left(\frac{\partial E_{k}}{\partial \dot{\theta}_{1}}\right)=\vec{l}_{2} m_{2}\left(\ddot{\theta}_{1}+\ddot{\theta}_{2}\right)+4\left(\ddot{\theta}_{1}+\ddot{\theta}_{2}\right) \dot{\theta}_{2} \vec{l}_{1} \vec{l}_{2} m_{2} \sin \theta_{2} \cos \theta_{2}+4\left(\ddot{\theta}_{1}\right.$

$\left.+\ddot{\theta}_{2}\right) \vec{l}_{4} m_{3} \sin ^{2}\left(\theta_{1}+\theta_{2}\right)+4\left(\dot{\theta}_{1}+\dot{\theta}_{2}\right)^{3} \vec{l}_{4} m_{3} \cos \left(\theta_{1}+\theta_{2}\right)+2\left(\dot{\theta}_{1}+\dot{\theta}_{2}\right) \vec{l}_{4}$

$m_{3} \sin ^{2}\left(\theta_{1}+\theta_{2}\right)+4\left(\dot{\theta}_{1}+\dot{\theta}_{2}+\dot{\theta}_{3}\right) \vec{l}_{4} m_{3} \sin \left(\theta_{1}+\theta_{2}+\theta_{3}\right)-{ }_{2} \dot{\theta}^{2} \vec{l}_{1} \vec{l}_{2} m_{2}$

$\sin \theta_{2}-2\left(\dot{\theta}_{1}+\dot{\theta}_{2}\right) \dot{\theta}_{2} \vec{l}_{1} \vec{l}_{2} m_{2} \sin \theta_{1}+2 \ddot{\theta}_{l_{1}} \vec{l}_{2} m_{2} \cos \theta_{2}-{ }_{2} \dot{\theta}^{2} \vec{l}_{1} \vec{l}_{2} m_{2}$

$\left.\sin \theta_{2}+\ddot{\theta}_{l_{1}} \vec{l}_{2} m_{3} \cos \left(\theta_{1}+\theta_{2}\right)-\dot{\theta}_{1} \vec{l}_{1} \vec{l}_{2} m_{3} \dot{\theta}_{1}+\dot{\theta}_{2}\right) \sin \left(\theta_{1}+\theta_{2}\right)+\vec{l}_{2}$

$\left.m_{3}\left(\ddot{\theta}_{1}+\ddot{\theta}_{2}+\ddot{\theta}_{3}\right) \cos \left(\theta_{1}+\theta_{2}\right)+\vec{l}_{2} m_{3} \dot{\theta}_{1}+\dot{\theta}_{2}+\dot{\theta}_{3}\right)\left(\dot{\theta}_{1}+\dot{\theta}_{2}\right) \cos \left(\theta_{1}+\theta_{2}\right.$

)$\left.+\vec{l}_{2} m_{3}\left(\ddot{\theta}_{1}+\ddot{\theta}_{2}\right) \cos \theta_{3}+\vec{l}_{2} m_{3} \dot{\theta}_{1}+\dot{\theta}_{2}\right) \dot{\theta}_{3} \cos \theta_{3}+\vec{l}_{1} \vec{l}_{2} m_{3} \ddot{\theta}_{1}+\ddot{\theta}_{2}+\ddot{\theta}_{3}$

) $\cos \theta_{3}+\vec{l}_{2} \vec{l}_{3} m_{3}\left(\ddot{\theta}_{1}+\ddot{\theta}_{2}+\ddot{\theta}_{3}\right) \cos \theta_{3}+\vec{l}_{2} \vec{l}_{3} m_{3}\left(\dot{\theta}_{1}+\dot{\theta}_{2}+\dot{\theta}_{3}\right) \dot{\theta}_{3}+4\left(\ddot{\theta}_{1}+\right.$

$\left.\ddot{\theta}_{2}\right) \vec{l}_{1} \vec{l}_{4}+m_{3} \cos \left(\theta_{1}+\theta_{2}\right)+2\left(\dot{\theta}_{1}+\dot{\theta}_{2}\right)^{3} \vec{l}_{1} \vec{l}_{4}+m_{3} \cos \left(\theta_{1}+\theta_{2}\right)-2\left(\dot{\theta}_{1}+\dot{\theta}_{2}+\dot{\theta}_{3}\right.$

$\vec{l}, \vec{l}_{4} m_{3} \sin \left(\theta_{1}+\theta_{2}+\theta_{3}\right)$

$\frac{d}{d t}\left(\frac{\partial E_{k}}{\partial \dot{\theta}_{2}}\right)=\vec{l}_{2} m_{2}\left(\ddot{\theta}_{2}+\ddot{\theta}_{1}\right)+2 \vec{l}_{4} m_{3}\left(\dot{\theta}_{1}+\dot{\theta}_{2}\right)\left(\ddot{\theta}_{2}+\ddot{\theta}_{1}\right) \sin ^{2}\left(\theta_{1}+\theta_{2}\right)-4 \vec{l}_{4} m_{3}\left(\dot{\theta}_{1}\right.$

$\left.+\dot{\theta}_{2}\right)^{2}\left(\ddot{\theta}_{2}+\ddot{\theta}_{1}\right) \sin \left(\theta_{1}+\theta_{2}\right) \cos \left(\theta_{1}+\theta_{2}\right)+2 \vec{l}_{4} m_{3}\left(\ddot{\theta}_{1}+\ddot{\theta}_{2}+\ddot{\theta}_{3}\right)\left(\dot{\theta}_{1}+\dot{\theta}_{2}\right) \sin ^{2}\left(\theta_{1}\right.$ $\left.+\theta_{2}\right)+2 \vec{l}_{4} m_{3}\left(\ddot{\theta}_{1}+\ddot{\theta}_{2}\right) \sin ^{2}\left(\theta_{1}+\theta_{2}\right)-4 \vec{l}_{4} m_{3} \dot{\theta}_{1}\left(\dot{\theta}_{1}+\dot{\theta}_{2}\right)^{2} \sin \left(\theta_{1}+\theta_{2}\right) \cos \left(\theta_{1}+\right.$

$\left.\theta_{2}\right)+2 \vec{l}_{1} \vec{l}_{2} m_{3} \ddot{\theta}_{1} \cos \theta_{2}+2 \vec{l}_{1} \vec{l}_{2} m_{2} \dot{\theta}_{1} \dot{\theta}_{2} \cos \theta_{2}+\vec{l}_{1} \vec{l}_{2} m_{3}\left(\ddot{\theta}_{1}+\ddot{\theta}_{2}\right) \cos \left(\theta_{1}+\theta_{2}\right)$

$-\vec{l}_{1} \vec{l}_{2} m_{3}\left(\dot{\theta}_{1}+\dot{\theta}_{2}+\dot{\theta}_{3}\right)\left(\dot{\theta}_{1}+\dot{\theta}_{2}\right) \sin \left(\theta_{1}+\theta_{2}\right)+\vec{l}_{1} \vec{l}_{2} m_{3}\left(\ddot{\theta}_{1}+\ddot{\theta}_{2}+\ddot{\theta}_{3}\right) \cos \theta_{1}-\vec{l}_{1} \vec{l}_{2}$

$m_{3}\left(\dot{\theta}_{1}+\dot{\theta}_{2}+\dot{\theta}_{3}\right) \dot{\theta}_{1} \sin \theta_{1}+\vec{l}_{2} \vec{l}_{3} m_{3}\left(\ddot{\theta}_{1}+\ddot{\theta}_{2}+\ddot{\theta}_{3}\right) \cos \theta_{3}+2 \vec{l}_{1} \vec{l}_{4} m_{3}\left(\dot{\theta}_{1}+\dot{\theta}_{2}\right) \cos \left(\theta_{1}\right.$

$\left.+\theta_{2}\right)+2 \vec{l}_{1} \vec{l}_{4} m_{3}\left(\dot{\theta}_{1}+\dot{\theta}_{2}+\dot{\theta}_{3}\right) \cos \left(\theta_{1}+\theta_{2}\right)+2 \vec{l}_{1} \vec{l}_{4} m_{3}\left(\dot{\theta}_{1}+\dot{\theta}_{2}+\dot{\theta}_{3}\right) \cos \left(\theta_{1}+\theta_{2}+\theta_{3}\right)$ 


$$
\begin{aligned}
& \frac{d}{d t}\left(\frac{\partial_{E_{k}}}{\partial \dot{\theta}_{3}}\right)=\vec{l}_{1} \vec{l}_{2} m_{3} \ddot{\theta}_{1}\left(\dot{\theta}_{1}+\dot{\theta}_{2}+\dot{\theta}_{3}\right)+2 \vec{l}_{4} m_{3}\left(\ddot{\theta}_{1}+\ddot{\theta}_{2}+\ddot{\theta}_{3}\right) \sin { }^{2}\left(\theta_{1}+\theta_{2}+\theta_{3}\right)+ \\
& 4 \vec{l}_{4} m_{3}\left(\dot{\theta}_{1}+\dot{\theta}_{2}+\dot{\theta}_{3}\right)^{2} \sin \left(\theta_{1}+\theta_{2}+\theta_{3}\right) \cos \left(\theta_{1}+\theta_{2}+\theta_{3}\right)-\vec{l}_{1} \vec{l}_{2} m_{3}\left(\ddot{\theta}_{1}+\ddot{\theta}_{2}+\ddot{\theta}_{3}\right)( \\
& \left.\dot{\theta}_{1}+\dot{\theta}_{2}\right) \sin \left(\theta_{1}+\theta_{2}\right)+\vec{l}_{1} \vec{l}_{2} m_{3} \ddot{\theta}_{1} \cos \left(\theta_{1}+\theta_{2}\right)+\vec{l}_{1} \vec{l}_{2} m_{3} \ddot{\theta}_{1} \cos \left(\theta_{1}+\theta_{2}\right)+\vec{l}_{3} \vec{l}_{2} m_{3} \\
& \left(\ddot{\theta}_{1}+\ddot{\theta}_{3}\right) \cos \theta_{3}-\vec{l}_{2} \vec{l}_{3} m_{3}\left(\dot{\theta}_{1}+\dot{\theta}_{3}\right) \dot{\theta}_{3} \sin \theta_{3}-\vec{l}_{1} \vec{l}_{4} m_{3} \cos \left(\dot{\theta}_{1}+\dot{\theta}_{2}+\dot{\theta}_{3}\right) \\
& \frac{\partial_{E_{k}}=\vec{l}_{1}\left(\vec{l}_{1}+\vec{l}_{4}+\vec{l}_{5}\right)\left(m_{1}+m_{2}+m_{3}\right) \theta_{1}+\vec{l}_{2}\left(\vec{l}_{3}+\vec{l}_{4}+\vec{l}_{5}\right) m_{2}\left(\theta_{1}+\theta_{2}\right)+\vec{l}_{3}\left(\vec{l}_{3}+\vec{l}_{4}+\vec{l}_{5}\right) m_{3}\left(\theta_{1}+\theta_{2}+\theta_{3}\right)}{\partial \theta_{1}} \\
& +2 \vec{l}_{4} m_{5} \dot{\theta}_{1}\left(\dot{\theta}_{1}+\dot{\theta}_{2}\right)^{2} \sin \left(\theta_{1}+\theta_{2}\right)-2 \vec{l}_{4} m_{5} \dot{\theta}_{1}\left(\dot{\theta}_{1}+\dot{\theta}_{2}+\dot{\theta}_{3}\right)^{2} \sin \left(\theta_{1}+\theta_{2}+\theta_{3}\right)-\vec{l}_{1} \vec{l}_{2} m_{2} \dot{\theta}_{1}\left(\dot{\theta}_{1}+\dot{\theta}_{2}+\dot{\theta}_{3}\right) \sin \\
& \left(\theta_{1}+\theta_{2}\right)+\left(\vec{l}_{4}+\vec{l}_{5}\right)\left(m_{2}+m_{2}+m_{3}\right) \theta_{1}+\left(\vec{l}_{4}+\vec{l}_{5}\right) m_{2}\left(\theta_{1}+\theta_{2}\right)+2 \vec{l}_{1} \vec{l}_{4} m_{3}\left(\dot{\theta}_{1}+\dot{\theta}_{2}\right) \cos \left(\theta_{1}+\theta_{2}\right)+2 \vec{l}_{1} \vec{l}_{4} m_{3} \\
& \left.\dot{\theta}_{1}+\dot{\theta}_{2}+\dot{\theta}_{3}\right) \cos \left(\theta_{1}+\theta_{2}\right)
\end{aligned}
$$

$\frac{\partial E_{K}}{\partial \theta_{2}}=\vec{l}_{2}\left(\vec{l}_{2}+\vec{l}_{4}+\vec{l}_{5}\right) m_{2}\left(\theta_{1}+\theta_{2}\right)+\vec{l}_{3}\left(\vec{l}_{3}+\vec{l}_{4}+\vec{l}_{5}\right) m_{3}\left(\theta_{1}+\theta_{2}+\theta_{3}\right)+4 \vec{l}_{4} m_{3} \dot{\theta}_{1} \sin \theta_{2}+2 \vec{l}_{4} m_{3} \dot{\theta}_{1}\left(\dot{\theta}_{1}+\dot{\theta}_{2}\right)^{2} \sin$ $\left.\left(\theta_{1}+\theta_{2}\right)+2 \vec{l}_{4} m_{3} \dot{\theta}_{1}\left(\dot{\theta}_{1}+\dot{\theta}_{2}+\dot{\theta}_{3}\right)^{2} \sin \left(\theta_{1}+\theta_{2}+\theta_{3}\right)-2 \vec{l}_{1} \vec{l}_{2} m_{3} \dot{\theta}_{1}\left(\dot{\theta}_{1}+\dot{\theta}_{2}\right) \sin \theta-2 \vec{l}_{1} \vec{l}_{2} m_{3} \dot{\theta}_{1} \dot{\theta}_{1}+\dot{\theta}_{2}\right) \sin \theta-\vec{l}_{2}$ $\left.+\vec{l}_{5}\right) m_{3} \dot{\theta}_{1}\left(\theta_{1}+\theta_{2}\right)+2 \vec{l}_{1} \vec{l}_{4} m_{3} \dot{\theta}_{1}\left(\dot{\theta}_{1}+\dot{\theta}_{2}+\dot{\theta}_{3}\right) \cos \left(\theta_{1}+\theta_{2}\right)-\left(\vec{l}_{4}+\vec{l}_{5}\right) m_{3} \dot{\theta}_{1}\left(\theta_{1}+\theta_{2}\right)+2 \vec{l}_{1} \vec{l}_{4} m_{3} \dot{\theta}_{1} \cos \left(\theta_{1}+\theta_{2}\right)+$ $2 \vec{l}_{3} \vec{l}_{4} m_{3}\left(\dot{\theta}_{1}+\dot{\theta}_{1}+\dot{\theta}_{2}\right) \cos \left(\theta_{1}+\theta_{2}+\theta_{3}\right)$

$$
\begin{aligned}
& \frac{\partial E_{K}}{\partial \theta_{3}}=\vec{l}_{3}\left(\vec{l}_{3}+\vec{l}_{4}+\vec{l}_{5}\right)\left(\theta_{1}+\theta_{2}+\theta_{3}\right)-2 \vec{l}_{4} m_{3}\left(\dot{\theta}_{1}+\dot{\theta}_{2}+\dot{\theta}_{3}\right)^{2} \sin \left(\theta_{1}+\theta_{2}+\theta_{3}\right)-\vec{l}_{2} \vec{l}_{3} m_{3}\left(\dot{\theta}_{1}+\dot{\theta}_{2}\right) \\
& \left(\dot{\theta}_{1}+\dot{\theta}_{2}+\dot{\theta}_{3}\right) \sin \theta_{3}-2 \vec{l}_{3} \vec{l}_{4} m_{3}\left(\dot{\theta}_{1}+\dot{\theta}_{2}+\dot{\theta}_{3}\right) \cos \left(\theta_{1}+\theta_{2}+\theta_{3}\right)
\end{aligned}
$$

\section{potential energy of System}

$$
E_{P}=\left(\vec{l}_{1}+\vec{l}_{4}\right) m_{1} g \cos \theta_{1}+\left(\vec{l}_{2}+\vec{l}_{4}\right) m_{2} g \cos \left(\theta_{1}+\theta_{2}\right)+\left(\vec{l}_{3}+\vec{l}_{4}\right) m_{3} g \cos \left(\theta_{1}+\theta_{2}+\theta_{3}\right)
$$

(18)

$$
\frac{\partial E_{p}}{\partial \theta}=\vec{l}_{1} \vec{l}_{4} m_{1} g \dot{\theta}_{1} \sin \theta
$$




$$
\begin{aligned}
& \frac{\partial E_{p}}{\partial \theta}=\vec{l}_{2} \vec{l}_{4} m_{2} g \dot{\theta}_{2} \sin \left(\theta_{1}+\theta_{2}\right) \\
& \frac{\partial E_{p}}{\partial \theta}=\vec{l}_{3} \vec{l}_{4} m_{3} g \dot{\theta}_{3} \sin \left(\theta_{1}+\theta_{2}+\theta_{3}\right)
\end{aligned}
$$

Substituting Lagrange equation below (10) for above equations

Lagrange equation is

$$
\frac{d}{d t}\left(\frac{\partial_{E_{\kappa}}}{\partial \dot{q}_{i}}\right)-\frac{\partial_{E_{\kappa}}}{\partial q_{i}}+\frac{\partial_{E_{p}}}{\partial q_{i}}=F_{i},(\mathrm{i}=1,2, \ldots, \mathrm{n})
$$

Here $E_{\kappa}$ is kinetic of system;

$E_{p} \quad$ is potential energy of system;

$q_{i}$ is generalized coordinate, it is a group of independent parameters that can define mechanical system movement;

$F_{i}$ is generalized force, when $q_{i}$ is a angular displacement it a torque, when $q_{i}$ is linear displacement it a force;

$\mathrm{n}$ is system generalized coordinate.

System generalized force

Supposed that $\operatorname{Fk}(\mathrm{k}=1,2, . ., \mathrm{m})$ and $\mathrm{Mj}(\mathrm{j}=1,2, . ., \mathrm{n})$ is force and torque acting on system. Its power is

$$
P=\sum_{k=1}^{m}\left(F_{k} v_{k} \cos \alpha_{k}\right)+\sum_{j=1}^{n}\left( \pm_{M} \omega_{j}\right)
$$

Here $\omega \mathrm{j}$ : angular velocity acting on component with $\mathrm{Mj}$;

vk: the velocity in force Fk point of action; (the syntropy + , reverse direction -)

ak: angle between Fk and vk

When generalized coordinates is $\varphi$ angular displacement generalized force=equivalent torque Me. 


$$
\delta_{W_{2}}=\sum\left(F_{k} \delta_{v_{k}} \cos \alpha_{k}\right)+\sum\left({ }_{M}{ }_{M} \delta \omega_{j}\right)
$$

Here ${ }^{a_{k}}$ is zero; $\quad F_{k}=200 \mathrm{~N} ;{ }^{{ }_{k}}=0.2 \sim 0.3 \mathrm{~m} / \mathrm{s} ;{ }^{\omega}=; 20 \sim 30^{\circ} / \mathrm{s}^{M}=20 \sim 30 \mathrm{Nm}$. ${ }^{\delta \varphi}$ is virtual

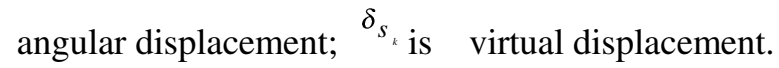

Supposing that

$$
\begin{gathered}
\delta_{S_{K}}=\frac{\partial_{s_{K}}}{\partial q_{1}} \delta_{q_{1}}+\frac{\partial_{s_{K}}}{\partial q_{2}} \delta_{q_{2}} \\
\delta \varphi_{K}=\frac{\partial \varphi_{j}}{\partial q_{1}} \delta_{q_{1}}+\frac{\partial \varphi_{j}}{\partial q_{2}} \delta_{q_{2}}
\end{gathered}
$$

Replace equation below with above two equations

$$
\left\{\begin{array}{l}
F_{1}=\sum_{k=1}^{m}\left[F_{k} \frac{\partial_{s_{k}}}{\partial_{q_{1}}} \cos \alpha_{k}\right]+\sum_{j=1}^{k}\left[M, \frac{\partial \varphi_{j}}{\partial_{q_{1}}}\right] \\
F_{2}=\sum_{k=1}^{m}\left\lfloor F_{k} \frac{\partial_{s_{k}}}{\partial_{q_{2}}} \cos \alpha_{k}\right]+\sum_{j=1}^{\infty}\left[M, \frac{\partial \varphi_{j}}{\partial_{q_{2}}}\right\rfloor
\end{array}\right.
$$

This is generalized force equation.

\section{Conclusions}

In the modeling of five freedoms in hammer of robotic arm the kinetic equation is established according to Lagrange equation based on two freedoms robotic arm. It compensates the blank in three freedoms and one impulsion on robotic arm. It is found that the first and second item is complicated and long besides others is concise. Referring to the important occasion the kinetic equation will only be computed on three freedoms according to this study.

\section{References}

[1] Zhang ce. Machinery dynamics. Higher Educational Press, 2008: 96 
[2] Li Doyi. Chen Lei, Shang Xiaolong, Wang Zhinchao, Wang Wei, Yang Xulong. Structrue design of pineapple picking manipulator[J], Agricultural Engineering. 2019, 9(2): 1 2

\section{Brief Biography}

XU Run is a PHD in school of Material Engineering Department in Geongsang National University. His research direction is material of metal cutting\& management and business administration etc. Xurun1206@163.com 
Figures

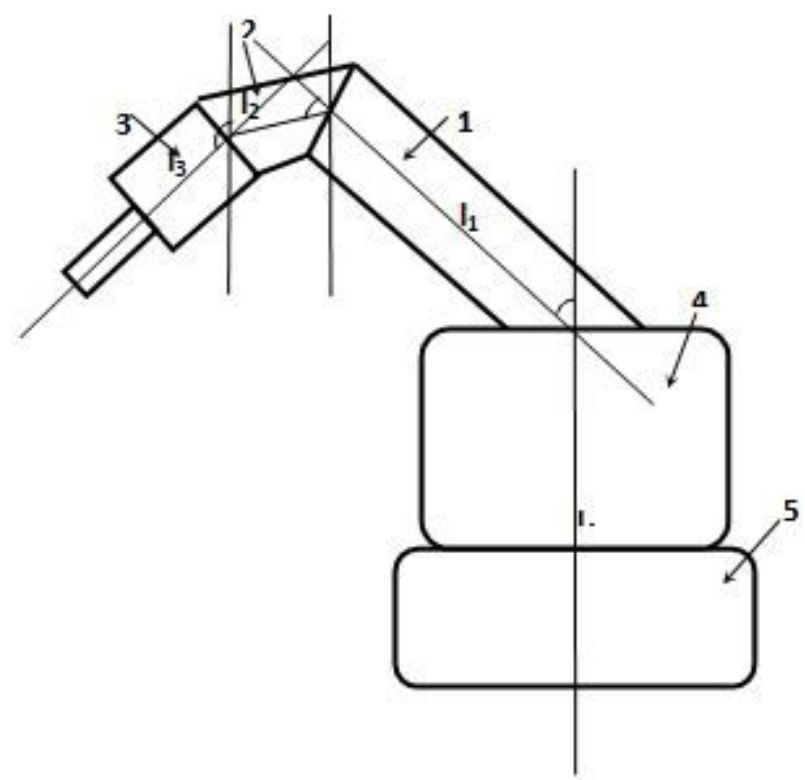

Figure 1

construction schematic of mechanical arm in series in robot 


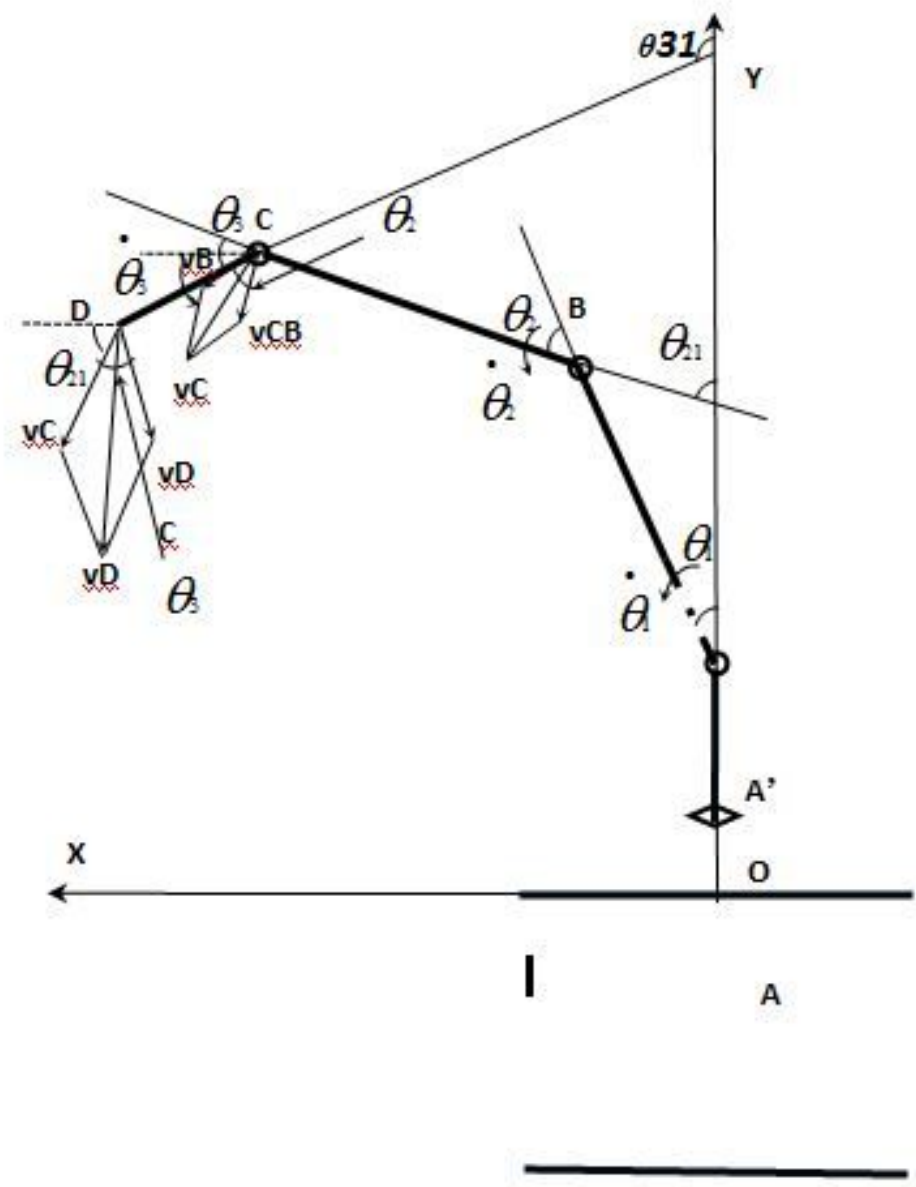

Figure 2

principle schematic of mechanical arm in series in robot 